\title{
The widely used SPECT and PET tracers for cardiac sympathetic nervous system
}

\author{
Zhiping Shu and Xiaohua Zhu* \\ Department of Nuclear Medicine, Tongji Hospital, Tongji Medical College, Huazhong University of Science and Technology, 1095 Jie Fang Avenue, Wuhan 430030, China.
}

\begin{abstract}
Dysfunction of the cardiac nervous system implicated in various types of cardiac disease has been recognized. With the development of single photon emission computed tomography (SPECT) and positronemission tomography (PET), nuclear imaging technique make it possible for global and regional investigation of the myocardial nervous system. The SPECT tracer ${ }^{123} \mathrm{I}$-metaiodobenzy lguanidine $\left({ }^{123} \mathrm{I}-\mathrm{MIBG}\right)$ has been applied for mapping presynaptic sympathetic innervation and is available for imaging on various heart diseases now. PET tracer ${ }^{11} \mathrm{C}$-meta-hydroxyephedrine $\left({ }^{11} \mathrm{C}-\mathrm{HED}\right)$ has also been established for characterizing the cardiac sympathetic nervous system. And the PET tracer LMI1195, which is under clinical investigation, is a promising tracer.
\end{abstract}

\begin{abstract}
Abbreviations: SPECT: single photon emission computed tomography, PET: positronemission tomography, DOPA: dihydroxyphenylalanine, MOA: monoamine oxidase, MIBG: metaiodobenzylguanidine, CAD: coronary artery disease
\end{abstract}

\section{Cardiac sympathetic nervous system}

The cardiac nervous system was divided into two portions: sympathetic and parasympathetic innervation. Among the cardiac nervous system, the sympathetic nervous system is the critical autonomic component in the ventricles, whereas parasympathetic nerve fibers are primary in the atria and less abundant in ventricular myocardium. Autonomic nerves travel along coronary vascular structures and go into underlying myocardium. NE concentration shows a gradient in sympathetic innervation from the base to the apex of the heart $[1,2]$. Norepinephrine and acetylcholine are their major neurotransmitters respectively, which binding to adrenergic receptors and muscarinic or neuronal nicotinic receptors at target cardiac tissue. The two neurotransmitters exert stimulating or inhibitory effects on cardiac inotropy and chronotropy (force of contraction, heart rate and conduction) via adrenergic and muscarinic receptors to adapt to changing cardiovascular demand. In all, the cardiac neuronal network exerting a reciprocal, concerted action plays an important role in the performance of cardiovascular system. The pathophysiology of cardiac disease is attributed to the dysfunction of the cardiac nervous system.

Inneuronalvesicles, tyrosineisconverted to dihydroxyphenylalanine (DOPA), then with the help of DOPA-decarboxylase, the DOPA is further converted to dopamine. Dopamine $\beta$-hydroxylase catalyzes the dopamine into NE. Some NE is further transformed to epinephrine by phenylethanolamine-N-methyltransferase [3].And choline acetyltransferase catalyzes the choline and acetyl-CoA into ACh. Then, the release of NE and ACh into synaptic cleft is mainly regulated by sympathetic nerve and parasympathetic nerve respectively. The NE reuptake transporter (uptake-1) could return synaptic NE to the neuronal cytosol for packaging into vesicles or degradation by monoamine oxidase (MAO) and catechol-O-methyltransferase, as the cocaine and desipramine could pharmacologically blocked the process
[4-6]. Furthermore, more researches show that uptake-1 system density is highest in the left ventricle, less abundant in right ventricle and atrial tissue [7]. Only a small part of synaptic NE is subject to postsynaptic transport via the uptake-2 pathway (Figure 1) [8,9]. And a small proportion of the released norepinephrine also diffuses into vascular space [10]. Choline is recovered into the neuron to be reincorporated into Ach after ACh in the synapse is broken down by the acetylcholinesterase.

\section{${ }^{123}$ I-metaiodobenzylguanidine (MIBG)}

${ }^{123}$ I-metaiodobenzylguanidine(MIBG), as a single-photon emission computed tomography (SPECT)tracer, acts selectively on sympathetic nerve ending, that is an analogue of the antihypertensive drug guanethidine (Table 1) [11]. MIBG has been developed as the most widely used nuclear tracer for studying cardiac sympathetic activity in various of cardiac diseases. As a false neurotransmitter, MIBG is not metabolised by MAO or COMT, which allow it to be a nuclear tracer $[12,13]$. The uptake- 1 mechanism is responsible for transporting MIBG into neurons, so it also play a important role in the MIBG uptake. In addition to uptake-1 mechanism, the uptake-2 system removes MIBG into nonneuronal tissue [14-16]. It has been reported that the clearance of MIBG from the extraneuronal compartment is much faster than clearance from the neuronal tissues $[15,16]$. So MIBG uptake is not observed in denervated myocardium in transplanted heart [17].

After intravenous injection of 111 to $370 \mathrm{MBq}$ (3 to $10 \mathrm{mCi}$ ) ${ }^{123} \mathrm{I}-\mathrm{MIBG}$, early (15 to $30 \mathrm{~min}$ ) and late $(3$ to $4 \mathrm{~h}$ ) SPECT images obtained following the imaging protocol. By calculating a heart-to-

Correspondence to: Xiaohua Zhu, Department of Nuclear Medicine, Tongji Hospital, Tongji Medical College, Huazhong University of Science and Technology, 1095 JieFang Avenue, Wuhan430030, China, Tel: +86-2783663446; E-mail: evazhu@vip.sina.com

Key words: ${ }^{123} I-M I B G,{ }^{11} C-H E D,{ }^{18} F-L M I 1195$, Cardiac diseases, Parkinson disease.

Received: June 30, 2017; Accepted: July 26, 2017; Published: July 29, 2017 


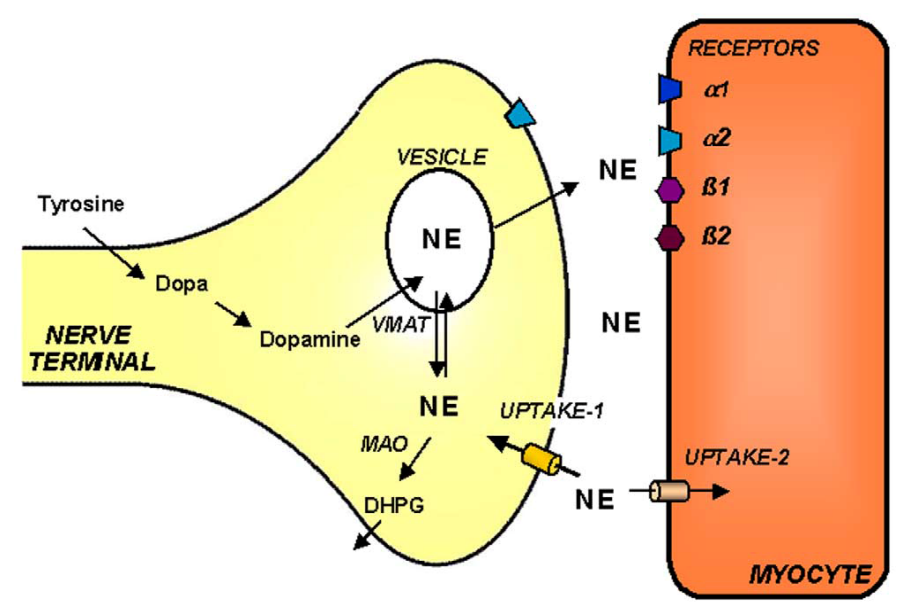

Uptake-1system; uptake-2 system; NE, Norepinephrine; VMAT, vesicular monoamine transporter; DHPG, dihydroxyphenylglycol.

Figure 1. Simplified schematic display of the cardiac sympathetic nervous system.

Table 1. Overview of radiotracers for sympathetic neurons.

\begin{tabular}{|l|l|l|}
\hline Type & Compound & Intraneuronal metabolism \\
\hline Catecholamine & F-18 (-)-6-fluoronorepinephrine & yes \\
\hline & F-18 6-fluorodopamine & yes \\
\hline $\begin{array}{l}\text { Catecholamine } \\
\text { analogue }\end{array}$ & C-11 epinephrine & yes \\
\hline & C-11 meta-hydroxyephedrine & no \\
\hline & C-11 phenylephrine & yes \\
\hline & F-18 6-fluorometaraminol & no \\
\hline & F-18 para-fluorobenzylguanidine & no \\
\hline & F-18 fluoroiodobenzylguanidine & no \\
\hline & $\begin{array}{l}\text { Br-76 } \\
\text { metabromobenzylguanidine }\end{array}$ & no \\
\hline & I-123 metaiodobenzylguanidine & no \\
\hline
\end{tabular}

mediastinum ratio (HMR) after drawing regions of interest over the heart and mediastinum, the heart MIBG uptake is semiquantified [18]. And by comparing early and delayed activities, the wash -out (WO) rate can be gained, which reflecting retention of NE by sympathetic neurons (Figure 2) [19]. Polar maps constructed from the SPECT images can evaluate the regional sympathetic activity. Differences between regional innervations and perfusion can be measured by comparing ${ }^{123} \mathrm{I}-\mathrm{MIBG}$ SPECT images with SPECT myocardial perfusion images [20,21]. Above all ${ }^{123}$ I-MIBG has been widely applied in cardiac diseases such as coronary artery diseases, myocardial infarction and heart failure.

\section{${ }^{11} \mathrm{C}-\mathrm{Hydroxyephedrine}$}

For the imaging of cardiac sympathetic neurons, the most widely used PET tracer is ${ }^{11} \mathrm{C}$-meta-hydroxyephedrine (HED). It is reliably synthesized in high specific activities by $\mathrm{N}$-methylation of the NE analogue metaraminol (Table 1). HED is resistant to metabolism by MAO or catechol-O-methyltransferase and transports through uptake-1 system [22]. Research in isolated perfused rat hearts after desipramine blockade and denervated human hearts demonstrated that nonspecific myocardial HED uptake is low $[23,24]$. Although vesicular storage seems to occur, binding inside vesicles is reduced because of a higher lipophilicity of HED compared with norepinephrine [25]. The cardiac HED retention index is largely relying on the norepinephrine transporter reuptake. When the additional desipramine or norepinephrine was added to the perfusate after use of HED in isolated rat hearts, the rat heart HED washout was accelerated. This phenomenon suggests that continuous release and reuptake of HED by sympathetic neurons is thus believed to undergoing [23]. With endogenous and exogenous catecholamines in isolated perfused rat hearts and in vivo in mice and humans, increased rate of tracer washout has been observed because of the competition for the uptake-1 system [26-29]. Treating with the VMAT inhibitor reserpine, the partial packaging of ${ }^{11} \mathrm{C}-\mathrm{HED}$ into vesicles by reducing tracer uptake was seen in dogs and rabbits researches [30]

Quantification of ${ }^{11} \mathrm{C}-\mathrm{HED}$ uptake is routinely reported as a retention index, a ratio of activity in the myocardium in the final image of a 40 or 60 minutes dynamic sequence to the integral of the imagederived arterial blood time activity curve [23,31]. As lower uptake of ${ }^{123}$ I- $m$ IBG was seen in the posterior inferior wall, distribution of HED throughout left ventricular myocardium in healthy normal individuals is consistent with other myocardial segments [24]. The retention of ${ }^{11} \mathrm{C}-\mathrm{HED}$, therefore, could reflect the whole spectrum of presynaptic activity, including uptake-1 system, vesicular packaging, and vesicular release.

\section{Coronary heart disease}

One of the primary clinical applications of sympathetic neuronal tracers was in the evaluation of patients with coronary artery disease. Compared with myocardial tissue, the sympathetic neuronal tissue is more easily damaged by the ischemia [32]. In patients with stable coronary artery disease without previous myocardial infarction, the reduction of MIBG uptake can be detected [33]. Research shows that the size of the MIBG defect was associated with severity of coronary stenoses, which demonstrated that cardiac adrenergic tissue is very sensitive to the damage caused by ischemia. In this study, the MIBG defect is significantly larger than MIBI defect when patients at rest and during exercise, which proves that the cardiac nerves is more vulnerable to the ischemia than the myocardial tissue [34]. But in another study, the overall diagnostic accuracy of late MIBG scanning was $66 \%$ and the positive and negative predictive values and kappa value were low; $60 \%, 70 \%$, and 0.31 , respectively [34]. Nonspecific reductions of MIBG uptake in the inferior and posterolateral regions restricted the diagnostic accuracy of MIBG tomography in coronary artery disease [34]. Researches show that although myocardial perfusion could be improved after 6 months exercise rehabilitation in patients with ischaemic heart disease, the size of innervation defects of myocardium did not show significant differences [35]. And ${ }^{123} I-M I B G$ could also be used for the examination of the patients incapable of exercising. Under normal circumstances, the perfusion imaging combined with pharmacological stress is a choice. However, some particular medical condition does not allow the use of pharmacological stress. Under the circumstances, resting imaging with ${ }^{123} \mathrm{I}-m \mathrm{IBG}$ combined with resting myocardial perfusion imaging (MPI) may be useful as a sign of reversible ischemia [35].

In patients after AMI, regional cardiac MIBG uptake in the region of infarction is significantly reduced. In all patients, the area of reduced MIBG uptake after 4 hours was more extensive than MIBG perfusion defect. And left ventricular ejection fraction correlated with the 4-hour MIBG defect score [36].

For the PET tracer ${ }^{11} \mathrm{C}-\mathrm{HED}$, the results of coronary artery disease are similary. In patients with coronary artery disease (CAD) without myocardial infarction, quantifiable ${ }^{11} \mathrm{C}-\mathrm{HED}$ and high resolution of PET could identify regional alterations in sympathetic innervation with normal resting perfusion. As to myocardial reinnervation after regional ischemic, the same to the MIBG, HED uptake of the myocardium is nearly unchanged in both diabetic and nondiabetic patients with stable 


\section{A}

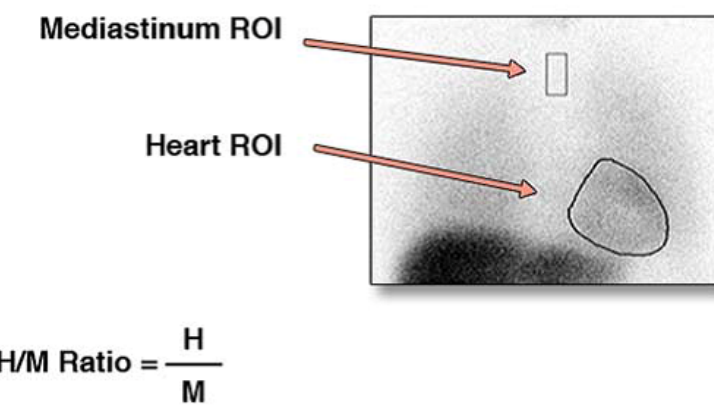

Heart ROI Mean Count: H

Mediastinum Mean Count: M

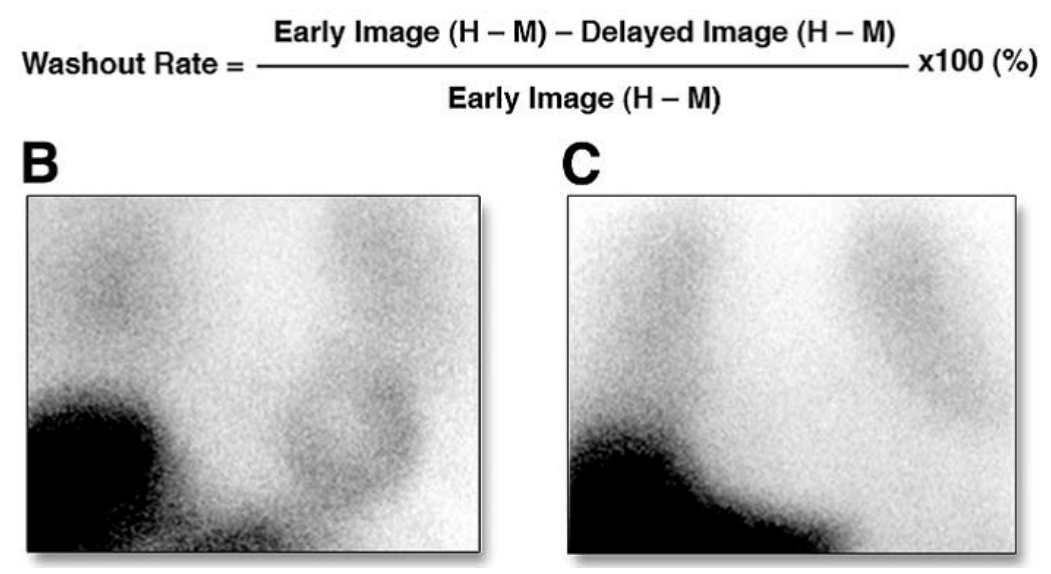

Figure 2. Calcution of Cardiac $m$ IBG Activity. (A) $m$ IBG heart-to-mediastinum ratio (HMR) and wash-out rate were calculated on an anterior view. (B) Normal cardiac $m$ IBG activity, a typical low-risk patient,with HMR: 1.70. (C) Severely decreased cardiac $m$ IBG activity, a typical high-risk patient, with HMR: 1.10.

chronic coronary artery disease in a 1-y follow-up [37]. These researches further supported the hypothesis of a higher sensitivity of sympathetic neurons to ischemia compared with cardiomyocytes [38]. As to the patients with an acute myocardial infarction, ${ }^{11} \mathrm{C}$ - hydroxyephedrine abnormalities were also more extensive than those for blood flow assessed by semiquantitative polar map analysis (Figure 3), especially in five patients with non-Q wave infarction [39]. And no change was also observed in regional reduction of HED uptake until 6 months after acute infarction, which demonstrated that there is no evidence of reinnervation of the injured myocardium [39]. In an animal study, to generate hibernating myocardium, pigs were underwent coronary artery restriction at the proximal left anterior descending artery. As a result, although significant functional improvement of hibernating myocardium owing to PCI or pravastatin therapy, no changes in HED defect size or severity was observed [40].

\section{Heart failure and cardiac mortality}

Cardiac remodeling is a hallmark among the development of cardiomyopathy and heart failure, which could result in the damage of sympathetic neurons. Enhanced ${ }^{123} I-M I B G$ washout rate and delayed but not early heart-to-mediastinal ratio(late HMR <1.74) can be observed in the patients with non-ischemic cardiomyopathy [41]. Also, the 11C-HED uptake permeability was significantly reduced among congestive heart failure patients $(\mathrm{LVEF}<45 \%)$ [42]. Regional defects of ${ }^{11} \mathrm{C}$-HED retention have been correlated with impaired contractile function and left ventricular ejection fraction, but not directly with oxidative metabolism [43-45]. Another research further demonstrate that depressed heart rate variability and baroreflex sensitivity, which consistent with impaired autonomic neuronal function, also has a close relationship with the reduction of ${ }^{11} \mathrm{C}-\mathrm{HED}$ in failing hearts [46].
${ }^{123}$ I-MIBG imaging has showed that abnormalities in global cardiac innervation could be a sign for cardiac mortality in patients with heart failure. A study including 112 heart failure patients with a poor LV function shows impaired cardiac sympathetic innervation imaged with ${ }^{123} \mathrm{I}-\mathrm{MIBG}$ correlated with poor prognosis. More research shows ${ }^{123} \mathrm{I}-\mathrm{MIBG}$ is the independent predictor for mortality, and compared with patients with reduced ${ }^{123} \mathrm{I}-M \mathrm{IBG}$, patients with normal ${ }^{123} \mathrm{I}-\mathrm{MIBG}$ showed a 2-year event-free survival [41,47]. As to ${ }^{11} \mathrm{C}-\mathrm{HED}$, the researches of PET imaging in chronic heart failure also shows that ${ }^{11} \mathrm{C}$-HED PET also provides independent prognostic information in patients with CHF [43].

\section{Arrhythmias}

Abnormalities in the autonomic nervous system have leaded to ventricular arrhythmias $[48,49]$. As cardiac sympathetic denervation has a close relationship with ventricular arrhythmias, sympathetic nerve imaging with ${ }^{123} \mathrm{I}-\mathrm{MIBG}$ could be used for predicting the occurrence of arrhythmias after myocardial infarction [50]. A pilot study that 17 patients suffering from sustained ventricular tachycardia or ventricular fibrillation treated with ICD showed apparently more global (early $\mathrm{H} / \mathrm{M}$ ratio) and regional sympathetic denervation (early and late ${ }^{123} \mathrm{I}-m$ IBG defect score) than patients without an ICD discharge (Figure 4) [51]. And advanced study demonstrated that late ${ }^{123} I-M I B G$ SPECT reflecting the regional cardiac sympathetic denervation was significantly correlated with ventricular arrhythmias needing appropriate ICD therapy. Moreover, late ${ }^{123} \mathrm{I}-\mathrm{MIBG}$ SPECT defect score could be an independent indicator for appropriate ICD therapy, but myocardial washout rate was not predictive for appropriate ICD therapy [52]. 

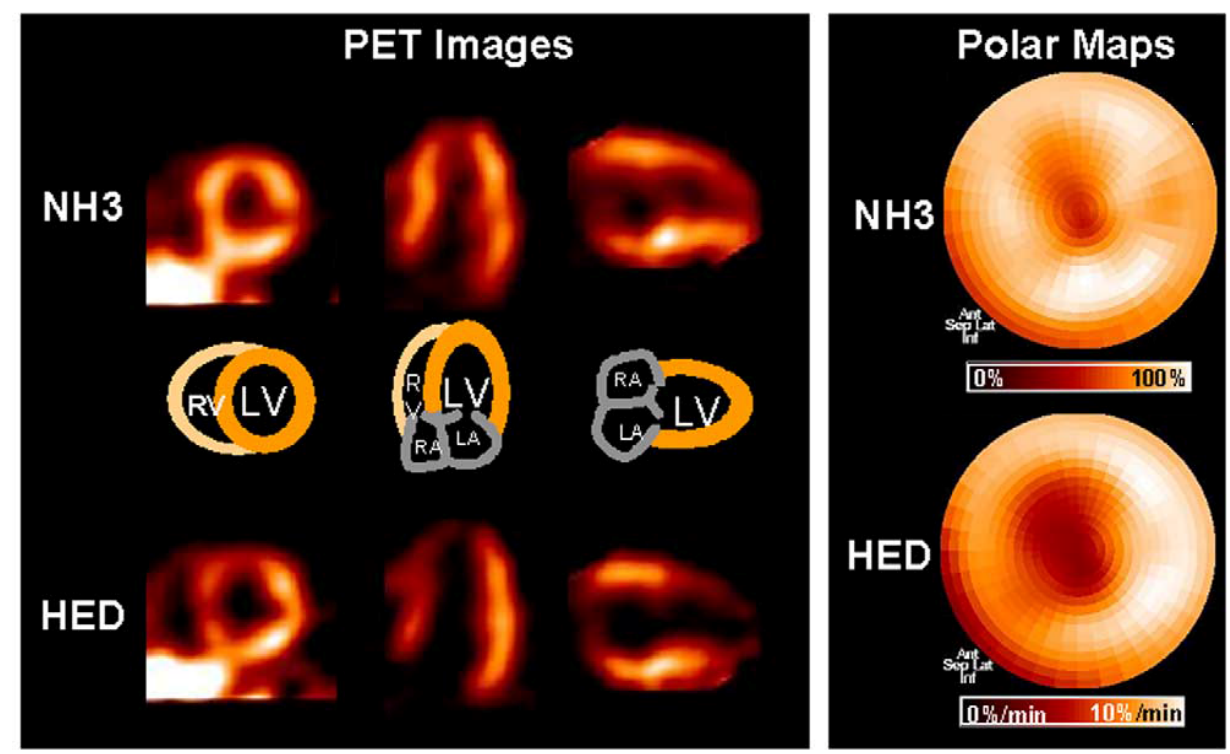

Figure 3. In a patient 3 weeks after nontransmural infarction of the distal left anterior descending artery territory. Mismatch of myocardial perfusion, as measured by nitrogen 13 ammonia $\left(\mathrm{NH}_{3}\right)$, and innervation, as measured by $\mathrm{C}-11$ hydroxyephedrine $(H E D)$.

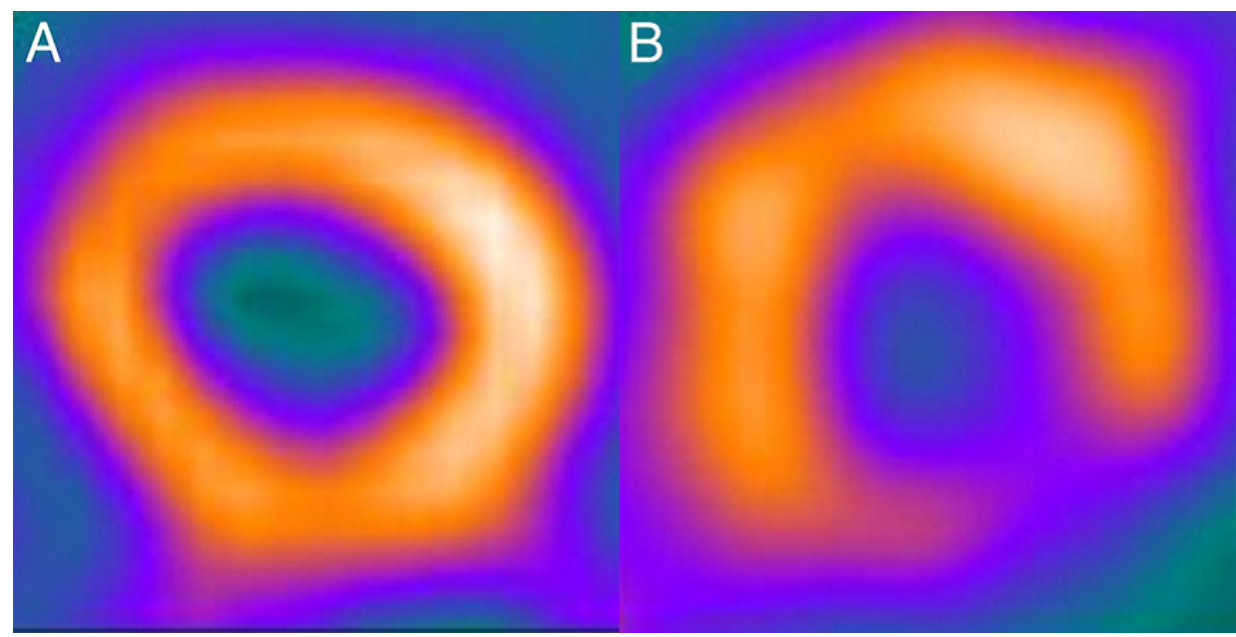

Figure 4. Example of Resting Myocardial Perfusion (A) and Late 123-I MIBG Imaging(B) in an ICD Patient.

Also abnormal patterns of ${ }^{11} \mathrm{C}-\mathrm{HED}$ retention were studied in right ventricular tachycardic arrhythmias. Patients with idiopathic right ventricular outflow tract tachycardia (RVO-VT) showed myocardial reduced HED uptake compared with the control subjects [53,54]. And in Brugada syndrome, compared with a group of age-matched healthy control subjects, the ${ }^{11} \mathrm{C}$-HED uptake, which representing presynaptic sympathetic norepinephrine recycling, was apparently increased in patients with Brugada syndrome [55]. In another study to evaluate cardiac sympathetic innervation with ${ }^{11} \mathrm{C}$-HED PET in genotyped LQTS patients, results show that decreased ${ }^{11} \mathrm{C}-\mathrm{HED}$ retention was seen in most LQTS patients [56].

\section{Parkinson disease}

As we all know that idiopathic Parkinson disease (IPD) is associated with multiple autonomic morbidities, including cardiac postganglionic and presynaptic sympathetic denervation. The PD patients imaged with ${ }^{123}$ I-MIBG demonstrate that cardiac sympathetic denervation is common. And the phenomenon seems to occur independent of the clinical stage and symptoms in patients with IPD, as the mean plasma adrenaline and noradrenaline levels are still in the normal level [57]. Cardiac sympathetic denervation imaged with ${ }^{123}$ I-MIBG has been proposed as a diagnostic test with which to differentiate IPD with postganglionic autonomic dysfunction from other Parkinsonian syndromes such as multiple system atrophy with central and preganglionic sympathetic dysfunction (Figure 5). In this research, significant positive relationship between cardiac MIBG uptake and the Cross-Cultural Smell Identification (CCSI) score in patients with PD could be found, whereas patients with MSA does not show significant correlation [58,59]. Primary progressive Freezing Gait (PPFG) also needs to distinguish with the Parkinson's Disease, because Freezing of gait (FOG) is a late manifestation of Parkinson's Disease. Results demonstrate that patients with PPFG show no reduction of cardiac MIBG uptake which could help distinguish patients with PPFG from those with PD and early FOG [60].

As cardiac PET is more sensitive and accurate than the SPECT, ${ }^{11} \mathrm{C}-\mathrm{HED}$ PET image is also used to reflect sympathetic nerve density 


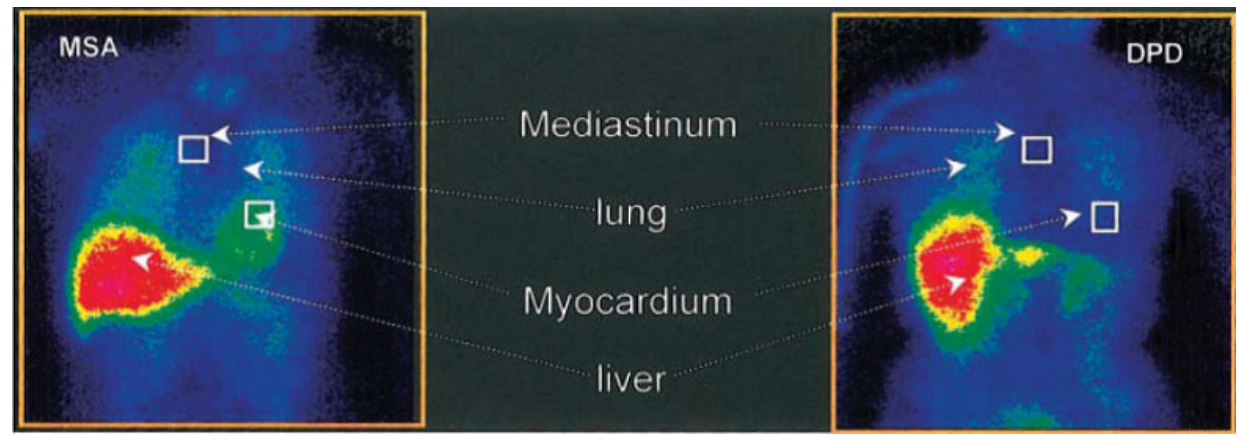

Figure 5. 120 mins after injection of ${ }^{123}$ I-MIBG in a patient with multiple system atrophy (MSA) and a patient with Parkinson's disease.

in PD patients. Compared with ${ }^{123} \mathrm{I}-\mathrm{MIBG}$ image, another research with the use of image with ${ }^{11} \mathrm{C}$ HED demonstrate that extensive cardiac denervation was observed in several patients with IPD [61,62]. However, among some patients with MSA and PSP, we could also observe the substantial cardiac denervation [62]. Furthermore whether cardiac sympathetic denervation in IPD inflences the left ventricle in a distinct regional pattern versus a more global pattern with application of ${ }^{11} \mathrm{C}$-HED PET was put into research. Results show that cardiac sympathetic denervation is extensive in IPD and affects most patients at an early stage in the disease course. The part of cardiac sympathetic denervation involves the proximal lateral left ventricular wall most severely, with relative sparing of the anterior and proximal septal walls [63].

\section{${ }^{18}$ F-LMI1195}

${ }^{18} \mathrm{~F}$-LMI1 195( ${ }^{18} \mathrm{~F}-\mathrm{N}$-[3-bromo-4-(3-fluoro-propoxy)-benzyl]guanidine) is a new PET tracer which is designed for imaging of cardiac sympathetic innervation. ${ }^{18} \mathrm{~F}$-LMI1195 can be synthesized by a singlestep nucleophilic substitution reaction, followed by a high performance liquid chromatography purification with a high radiochemical yield [64]. Although the ${ }^{11} \mathrm{C}-\mathrm{HED}$ and other PET tracers make it possible for assessment of cardiovascular sympathetic system abnormalities, the limitation of short half-life of ${ }^{11} \mathrm{C}(20 \mathrm{~min})$ reduces its clinical application because of the need for a costly on-site cyclotron [65].So the ${ }^{18} \mathrm{~F}$-LMI1195, a newly 18F-labeled PET imaging tracer has been developed to overcome these shortness of the PET tracers [64]. A longer physical half-life (110 $\mathrm{min})$ of $18 \mathrm{~F}$-radionuclides, allowing for delayed or prolonged imaging and more research plan. The in vitro assays showed that LMI1195 had a cell uptake profile similar to NE, comparable NET binding affinity and NET-mediated cell uptake kinetics [64].

The initial evaluation of this novel tracer was performed in cells and animal models. The uptake ratios of heart to adjacent organs such as blood, lung, and liver for LMI1195 were high in rabbits and higher than for ${ }^{123}$ I-MIBG in rats. In contrast to the high and sustained ${ }^{123}$ I-MIBG liver retention, these findings suggest that cardiac images of LMI1195 should have less interference by the background activity in organs such as the liver [64]. As discussed before, ${ }^{11} \mathrm{C}$-hydroxyephedrine cardiac retention is maintained by continuous reuptake via NET and release from the nerve terminals, further research show that ${ }^{18} \mathrm{~F}-\mathrm{LMI} 1195$, analogous to ${ }^{123} \mathrm{I}-\mathrm{MIBG}$, demonstrated stable tracer retention resistant to the NET inhibitor chase treatment, whereas $11 \mathrm{C}$-hydroxyephedrine cardiac retention is maintained by continuous reuptake via NET and release (diffusion) from the nerve terminals [66]. In rats, phenoxybenzamine but not desipramine could significantly inhibited cardiac uptake of ${ }^{18} \mathrm{~F}$-LMI1195, proving that $18 \mathrm{~F}$-LMI1195 is a substrate for the uptake- 2 mechanism and is consistent with the rat heart having a dominant level of the mechanism [67]. Also the first-inhuman study of the 18F-Labeled Tracer was conducted in 2014. 150$250 \mathrm{MBq}$ of LMI1195 were injected intravenously into twelve healthy subjects at 3 clinical sites. Then dynamic PET images were get over the heart for $10 \mathrm{~min}$, followed by sequential whole-body images for nearly $5 \mathrm{~h}$. No adverse events due to LMI1195 were found during the $5 \mathrm{~h}$. Blood radioactivity cleared quickly, whereas myocardial uptake remained stable and uniform throughout the heart over $4 \mathrm{~h}$. Favorable target-to-background ratios for cardiac imaging was obtained owing to rapidly clearance of liver and lung activity [68].

Impaired NET function in the heart has been identified in heart failure of animals and humans with different reseasons including hypertension, valvular and ischemic heart disease and idiopathic hypertrophic cardiomyopathy [69-71]. Therefore, measuring cardiac NET function may be useful for monitoring the disease progression and cardiac events in patients with HF. Imaging in these DSS rats showed reduced LMI1195 heart uptake following progression of HF development, as MIBG uptake was normal at the myocardial hypertrophy stage of DSS rats, even though the heart NE content decreased [72].

Regional cardiac sympathetic denervation (RCSD) associated with reduced noradrenaline transporter (NAT) function has been related to cardiac arrhythmia. LMI1195 cardiac imaging was applied for imaging of RCSD in a rabbit model surgically developed by regional phenol application on the left ventricular (LV) wall. So LMI1195 shows a close relationship with NAT and can be used for imaging RCSD [73].

As a promising PET nuclear tracer, the more clinical uses of LMI1195 in cardiac diseases need to be deeply studied. Advantages and disadvantages of LMI1195, compared with other PET tracers, also wait to be illustrated by researchers.

\section{Conclusion}

${ }^{123} \mathrm{I}-\mathrm{MIBG}$ and ${ }^{11} \mathrm{C}-\mathrm{HED}$ imaging provides a noninvasive tool for the investigation of cardiac sympathetic innervations which could be used for the assessments of various of cardiac diseases and Parkinson disease. As the sympathetic neuronal tissue is more easily damaged compared with the myocardium, so the ${ }^{123} \mathrm{I}-\mathrm{MIBG}$ and ${ }^{11} \mathrm{C}-\mathrm{HED}$ imaging is more sensitive than other examinations. But they are not commonly applied in patients with cardiac diseases or Parkinson disease. More clinical knowledge about cardiac sympathetic nervous system imaging needs further researches that define the prognostic importance of cardiac neuro imaging in various diseases. This may promote the licensure and deployment of neuronal imaging agents for routine use in the clinical setting. 


\section{Acknowledgment}

This project was supported by the National Natural Science Foundation of China (Grant No. 81271600 and Grant No. 81671718 Xiaohua Zhu)

\section{Disclosure}

The authors report no conflicts of interest.

\section{References}

1. Pierpont GL, DeMaster EG, Reynolds S, Pederson J, Cohn JN (1985) Ventricular myocardial catecholamines in primates. $J$ Lab Clin Med 106: 205-210. [Crossref]

2. Adams DJ, Cuevas J (2004) Electrophysiological properties of intrinsic cardiac neurons: the foundation of neurocardiology.

3. Francis GS (1988) Modulation of peripheral sympathetic nerve transmission. J Am Coll Cardiol 12: 250-254. [Crossref]

4. Jaques S, Tobes MC, Sisson JC (1987) Sodium dependency of uptake of norepinephrine and $\mathrm{m}$-iodobenzylguanidine into cultured human pheochromocytoma cells: evidence for uptake-one. Cancer research 47: 3920-3928.

5. Kopin IJ, Gordon EK (1963) Origin of Norepinephrine in the Heart. Nature 199: 1289. [Crossref]

6. Brüss M, Hammermann R, Brimijoin S, Bönisch H (1995) Antipeptide antibodies confirm the topology of the human norepinephrine transporter. Journal of Biological Chemistry 270: 9197-9201.

7. Wehrwein EA, Parker LM, Wright AA, et al. (2008) Cardiac norepinephrine transporter protein expression is inversely correlated to chamber norepinephrine content. American Journal of Physiology-Regulatory, Integrative and Comparative Physiology 295: R857-R863.

8. Russ H, Gliese M, Sonna J, Schömig E (1992) The extraneuronal transport mechanism for noradrenaline (uptake2) avidly transports 1-methyl-4-phenylpyridinium (MPP+). Naunyn-Schmiedeberg's archives of pharmacology 346: 158-165.

9. Salt $\mathrm{P}$ (1972) Inhibition of noradrenaline uptake 2 in the isolated rat heart by steroids, clonidine and methoxylated phenylethylamines. European journal of pharmacology 20: 329-340.

10. Esler M, Jennings G, Johns J, Burke F, Little P, et al. (1984) Estimation of'total'renal, cardiac and splanchnic sympathetic nervous tone in essential hypertension from measurements of noradrenaline release. Journal of hypertension Supplement 2: S123125.

11. Wieland DM, Wu J, Brown LE, Mangner TJ, Swanson DP, et al. (1980) Radiolabeled adrenergi neuron-blocking agents: adrenomedullary imaging with [131I] iodobenzylguanidine. J Nucl Med 21: 349-353. [Crossref]

12. Jaques S, Tobes M, Sisson J, Baker J, Wieland D (1984) Comparison of the sodium dependency of uptake of meta-lodobenzylguanidine and norepinephrine into cultured bovine adrenomedullary cells. Molecular pharmacology 26: 539-546.

13. Tobes MC, Jaques Jr S, Wieland DM, Sisson JC (1985) Effect of uptake-one inhibitors on the uptake of norepinephrine and metaiodobenzylguanidine. Journal of nuclear medicine 26: 897-907.

14. Glowniak JV, Kilty JE, Amara SG, Hoffman BJ, Turner FE (1993) Evaluation of metaiodobenzylguanidine uptake by the norepinephrine, dopamine and serotonin transporters. Journal of nuclear medicine 34: 1140-1146.

15. Nakajo M, Shimabukuro K, Yoshimura H (1986) Iodine-131 metaiodobenzylguanidine intra-and extravesicular accumulation in the rat heart. Journal of nuclear medicine 27 : $84-89$

16. Degrado TR, Zalutsky MR, Vaidyanathan G (1995) Uptake mechanisms of meta[123I]iodobenzylguanidine in isolated rat heart. Nucl Med Biol 22: 1-12. [Crossref]

17. Dae MW, De Marco T, Botvinick EH (1992) Scintigraphic assessment of MIBG uptake in globally denervated human and canine hearts--implications for clinical studies. Journal of nuclear medicine 33: 1444-1450.

18. Carrió I (2001) Cardiac neurotransmission imaging. J Nucl Med 42: 1062-1076. [Crossref]

19. Agostini D, Carrio I, Verberne HJ (2009) How to use myocardial 123I-MIBG scintigraphy in chronic heart failure. Eur J Nucl Med Mol Imaging 36: 555-559. [Crossref]
20. Estorch M, Carrió I, Berná L, López-Pousa J, Torres G (1995) Myocardial iodinelabeled metaiodobenzylguanidine 123 uptake relates to age. Journal of Nuclear Cardiology 2: 126-132.

21. Chen J, Garcia EV, Galt JR, Folks RD, Carrio I (2006) Optimized acquisition and processing protocols for I-123 cardiac SPECT imaging. Journal of nuclear cardiology 13: 251-260.

22. Raffel DM, Chen W (2004) Binding of [3H] mazindol to cardiac norepinephrine transporters: kinetic and equilibrium studies. Naunyn-Schmiedeberg's archives of pharmacology 370: 9-16.

23. DeGrado TR, Hutchins GD, Toorongian SA, Wieland DM, Schwaiger M (1993) Myocardial kinetics of carbon-11-meta-hydroxyephedrine: retention mechanisms and effects of norepinephrine. J Nucl Med 34: 1287-1293. [Crossref]

24. Schwaiger M, Kalff V, Rosenspire K (1990) Noninvasive evaluation of sympathetic nervous system in human heart by positron emission tomography. Circulation 82: 457 464

25. Raffel DM, Corbett JR, Del Rosario RB (1996) Clinical evaluation of carbon-11phenylephrine: MAO-sensitive marker of cardiac sympathetic neurons. Journal of Nuclear Medicine 37: 1923-1930.

26. Goldstein DS, Chang PC, Eisenhofer G (1990) Positron emission tomographic imaging of cardiac sympathetic innervation and function. Circulation 81: 1606-1621.

27. Nguyen NT, DeGrado TR, Chakraborty P, Wieland DM, Schwaiger M (1997) Myocardial kinetics of carbon-11-epinephrine in the isolated working rat heart. $\mathrm{J} \mathrm{Nucl}$ Med 38: 780-785. [Crossref]

28. Goldstein DS, Eisenhofer G, Dunn BB (1993) Positron emission tomographic imaging of cardiac sympathetic innervation using $6-[18 \mathrm{~F}]$ fluorodopamine: initial findings in humans. Journal of the American College of Cardiology 22: 1961-1971.

29. Del Rosario RB, Jung Y-W, Caraher J, Chakraborty PK, Wieland DM (1996) Synthesis and preliminary evaluation of [11 C]-(-)-phenylepnrine as a functional heart neuronal PET agent. Nuclear medicine and biology 23: 611-616.

30. Nomura Y, Matsunari I, Takamatsu H (2006) Quantitation of cardiac sympathetic innervation in rabbits using 11C-hydroxyephedrine PET: relation to 123I-MIBG uptake. European journal of nuclear medicine and molecular imaging 33: 871-878.

31. Caldwell JH, Kroll K, Li Z, Seymour K, Link JM, et al. (1998) Quantitation of presynaptic cardiac sympathetic function with carbon-11-meta-hydroxyephedrine. $J$ Nucl Med 39: 1327-1334. [Crossref]

32. Dae MW, O'Connell JW, Botvinick EH, Chin MC (1995) Acute and chronic effects of transient myocardial ischemia on sympathetic nerve activity, density, and norepinephrine content. Cardiovascular research 30: 270-280.

33. Hartikainen J, Mustonen J, Kuikka J, Vanninen E, Kettunen R (1997) Cardiac sympathetic denervation in patients with coronary artery disease without previous myocardial infarction. The American journal of cardiology 80: 273-277.

34. Nakata T, Nagao K, Tsuchihashi K, Hashimoto A, Tanaka S, et al. (1996) Regional cardiac sympathetic nerve dysfunction and the diagnostic efficacy of metaiodobenzylguanidine tomography in stable coronary artery disease. The American journal of cardiology 78: 292-297.

35. Estorch M, Flotats A, Serra-Grima R (2000) Influence of exercise rehabilitation on myocardial perfusion and sympathetic heart innervation in ischaemic heart disease. European journal of nuclear medicine 27: 333-339.

36. Matsunari I, Schricke U, Bengel FM (2000) Extent of cardiac sympathetic neurona damage is determined by the area of ischemia in patients with acute coronary syndromes. Circulation 101: 2579-2585.

37. Fricke E, Eckert S, Dongas A (2008) Myocardial sympathetic innervation in patients with symptomatic coronary artery disease: Follow-up after 1 year with neurostimulation. Journal of Nuclear Medicine 49: 1458-1464.

38. Bülow H, Stahl F, Lauer B (2003) Alterations of myocardial presynaptic sympathetic innervation in patients with multi-vessel coronary artery disease but without history of myocardial infarction. Nuclear medicine communications 24: 233-239.

39. Allman KC, Wieland DM, Muzik O, Degrado TR, Wolfe ER, et al. (1993) Carbon-11 hydroxyephedrine with positron emission tomography for serial assessment of cardiac adrenergic neuronal function after acute myocardial infarction in humans. Journal of the American College of Cardiology 22: 368-375.

40. Fallavollita JA, Banas MD, Suzuki G, Sajjad M, Canty Jr JM (2010) 11C-metahydroxyephedrine defects persist despite functional improvement in hibernating myocardium. Journal of nuclear cardiology 17: 85-96. 
41. Nakata T, Miyamoto K, Doi A (1998) Cardiac death prediction and impaired cardiac sympathetic innervation assessed by MIBG in patients with failing and nonfailing hearts. Journal of Nuclear Cardiology 5: 579-590.

42. Ungerer M, Hartmann F, Karoglan M (1998) Regional in vivo and in vitro characterization of autonomic innervation in cardiomyopathic human heart. Circulation 97: $174-180$.

43. Bengel F, Permanetter B, Ungerer M, Nekolla S, Schwaiger M (2001) Relationship between altered sympathetic innervation, oxidative metabolism and contractile function in the cardiomyopathic human heart; a non-invasive study using positron emission tomography. European heart journal 22: 1594-1600.

44. Bengel FM, Permanetter B, Ungerer M, Nekolla SG, Schwaiger M (2002) Alterations of the sympathetic nervous system and metabolic performance of the cardiomyopathic heart. European Journal of Nuclear Medicine and Molecular Imaging 29: 198-202.

45. Hartmann F, Ziegler S, Nekolla S (1999) Regional patterns of myocardial sympathetic denervation in dilated cardiomyopathy: an analysis using carbon- 11 hydroxyephedrine and positron emission tomography. Heart 81: 262-270.

46. Vesalainen RK, Pietilä M, Tahvanainen KU (1999) Cardiac positron emission tomography imaging with $[11 \mathrm{C}]$ hydroxyephedrine, a specific tracer for sympathetic nerve endings, and its functional correlates in congestive heart failure. The American Journal of Cardiology 84: 568-574.

47. Wakabayashi T, Nakata T, Hashimoto A (2001) Assessment of underlying etiology and cardiac sympathetic innervation to identify patients at high risk of cardiac death Journal of Nuclear Medicine 42: 1757-1767.

48. Mitrani RD, Klein LS, Miles WM (1993) Regional cardiac sympathetic denervation in patients with ventricular tachycardia in the absence of coronary artery disease. Journal of the American College of Cardiology 22: 1344-1353.

49. Bax JJ, Kraft O, Buxton AE (2008) 123I-mIBG Scintigraphy to Predict Inducibility of Ventricular Arrhythmias on Cardiac Electrophysiology Testing A Prospective Multicenter Pilot Study. Circulation: Cardiovascular Imaging 1: 131-140.

50. Yukinaka M, Nomura M, Ito S, Nakaya Y (1998) Mismatch between myocardial accumulation of 123 I-MIBG and $99 \mathrm{~m}$ Tc-MIBI and late ventricular potentials in patients after myocardial infarction: Association with the development of ventricular arrhythmias. American Heart Journal 136: 859-867.

51. Arora R, Ferrick KJ, Nakata T (2003) I-123 MIBG imaging and heart rate variability analysis to predict the need for an implantable cardioverter defibrillator. Journal of Nuclear Cardiology 10: 121-131.

52. Nagahara D, Nakata T, Hashimoto A (2008) Predicting the need for an implantable cardioverter defibrillator using cardiac metaiodobenzylguanidine activity together with plasma natriuretic peptide concentration or left ventricular function. Journal of Nuclear Medicine 49: 225-233.

53. Schäfers M, Lerch H, Wichter T (1998) Cardiac sympathetic innervation in patients with idiopathic right ventricular outflow tract tachycardia. Journal of the American College of Cardiology 32: 181-186.

54. Wichter T, Schäfers M, Rhodes CG (2000) Abnormalities of Cardiac Sympathetic Innervation in Arrhythmogenic Right Ventricular Cardiomyopathy Quantitative Assessment of Presynaptic Norepinephrine Reuptake and Postsynaptic B-Adrenergic Receptor Density With Positron Emission Tomography. Circulation 101: 1552-1558.

55. Kies P, Wichter T, Schäfers M (2004) Abnormal myocardial presynaptic norepinephrine recycling in patients with Brugada syndrome. Circulation 110: 3017-3022.

56. Mazzadi AN, André-Fouët X, Duisit J (2003) Cardiac retention of [11C] HED in genotyped long QT patients: a potential amplifier role for severity of the disease. American Journal of Physiology-Heart and Circulatory Physiology 285: H1286-H1293.
57. Rascol O, Schelosky L (2009) 123I-metaiodobenzylguanidine scintigraphy in Parkinson's disease and related disorders. Movement Disorders 24: S732-S741.

58. Shin DH, Lee PH, Bang OY, Joo IS, Huh K (2006) Clinical Implications of CardiacMIBG SPECT in the Differentiation of Parkinsonian Syndromes. J Clin Neurol 2: 51 57. [Crossref]

59. Kashihara K, Ohno M, Kawada S, Okumura Y (2006) Reduced cardiac uptake and enhanced washout of 123I-MIBG in pure autonomic failure occurs conjointly with Parkinson's disease and dementia with Lewy bodies. Journal of Nuclear Medicine 47: 1099-1101.

60. Salsone M, Bagnato A, Novellino F, Cascini GL, Paglionico S, et al. (2009) Cardiac MIBG scintigraphy in Primary Progressive Freezing Gait. Parkinsonism Relat Disord 15: 365-369. [Crossref]

61. Raffel DM, Koeppe RA, Little R, Wang CN, Liu S, et al. (2006) PET measurement of cardiac and nigrostriatal denervation in Parkinsonian syndromes. $J$ Nucl Med 47: 1769-1777. [Crossref]

62. Wong KK, Raffel DM, Koeppe RA, Frey KA, Bohnen NI, et al. (2012) Pattern of cardiac sympathetic denervation in idiopathic Parkinson disease studied with $11 \mathrm{C}$ hydroxyephedrine PET. Radiology 265: 240-247.

63. Berding G, Schrader CH, Peschel T (2003) [N-methyl 11C] meta-Hydroxyephedrine positron emission tomography in Parkinson's disease and multiple system atrophy. European Journal of Nuclear Medicine and Molecular Imaging 30: 127-131.

64. Yu M, Bozek J, Lamoy M (2011) Evaluation of LMI1195, a novel 18F-labeled cardiac neuronal PET imaging agent, in cells and animal models. Circulation: Cardiovascular Imaging 4: 435-443.

65. Fallavollita JA, Heavey BM, Luisi AJ (2014) Regional myocardial sympathetic denervation predicts the risk of sudden cardiac arrest in ischemic cardiomyopathy. Journal of the American College of Cardiology 63: 141-149.

66. Werner RA, Rischpler C, Onthank D (2015) Retention Kinetics of the 18F-Labeled Sympathetic Nerve PET Tracer LMI1195: Comparison with 11C-Hydroxyephedrine and 123I-MIBG. Journal of Nuclear Medicine 56: 1429-1433.

67. Higuchi T, Yousefi BH, Kaiser F, Gärtner F, Rischpler C, et al. (2013) Assessment of the 18 F-labeled PET tracer LMI1 195 for imaging norepinephrine handling in rat hearts. J Nucl Med 54: 1142-1146. [Crossref]

68. Sinusas AJ, Lazewatsky J, Brunetti J (2014) Biodistribution and radiation dosimetry of LMI1195: first-in-human study of a novel 18F-labeled tracer for imaging myocardial innervation. Journal of Nuclear Medicine 55: 1445-1451.

69. Liang C-S, Fan T, Sullebarger J, Sakamoto S (1989) Decreased adrenergic neuronal uptake activity in experimental right heart failure. A chamber-specific contributor to beta-adrenoceptor downregulation. Journal of Clinical Investigation 84: 1267.

70. Backs J, Haunstetter A, Gerber SH (2001) The neuronal norepinephrine transporter in experimental heart failure: evidence for a posttranscriptional downregulation. Journal of Molecular and Cellular Cardiology 33: 461-472.

71. Buss SJ, Backs J, Kreusser MM, Hardt SE, Maser-Gluth C, et al. (2006) Spironolactone preserves cardiac norepinephrine reuptake in salt-sensitive Dahl rats. Endocrinology 147: 2526-2534. [Crossref]

72. Nozawa T, Igawa A, Yoshida N (1998) Dual-tracer assessment of coupling between cardiac sympathetic neuronal function and downregulation of B-receptors during development of hypertensive heart failure of rats. Circulation 97: 2359-2367.

73. Yu M, Bozek J, Lamoy M (2012) LMI1195 PET imaging in evaluation of regional cardiac sympathetic denervation and its potential role in antiarrhythmic drug treatment European Journal of Nuclear Medicine and Molecular Imaging 39: 1910-1919.

Copyright: $(02017$ Shu Z. This is an open-access article distributed under the terms of the Creative Commons Attribution License, which permits unrestricted use, distribution, and reproduction in any medium, provided the original author and source are credited. 